\title{
El patrimonio inclusivo y la fabricación digital como herramientas de aprendizaje
}

\author{
PREMIO CIMED
}

\section{Inclusive heritage and digital fabrication as learning tools}

Versión en Español. *Full English Version at Anexo I

\begin{abstract}
Andrea Granell Querol ${ }^{\mathrm{a}}$ y Carme Comas Camacho ${ }^{\mathrm{b}}$
${ }^{a}$ Grupo de investigación Didáctica y Patrimonio DIDPATRI (Departamento de Didácticas Aplicadas de la Facultad de Educación, Universitat de Barcelona, agranellq@ub.edu) y ${ }^{\text {b}}$ Técnica de museos y accesibilidad, especializada en patrimonio cultural (Museos de Esplugues de Llobregat, ccomas@esplugues.cat)
\end{abstract}

\begin{abstract}
Resumen
Se presenta una propuesta de formación innovadora "Mirar con las manos" que, en cuatro bloques, pretende conocer el patrimonio local como herramienta de aprendizaje e inclusión social, a través de la fabricación digital y la reproducción 3D. El primer bloque, parte de la realidad del alumnado de secundaria quienes deben seleccionar el objeto personal que salvarian, y rellenar una ficha similar a las utilizadas en los inventarios de las colecciones museísticas. Este material sirve de base para la presentación teórica que, a través del diálogo y la reflexión sobre los objetos seleccionados, introduce los conceptos básicos sobre la importancia de conservar el patrimonio cultural como un pilar de nuestra identidad comunitaria. El segundo bloque trata la accesibilidad en los museos, presentando a los colectivos con más dificultades y qué recursos existen actualmente. Se trabaja sobre todo la accesibilidad en la comunicación, a través de la lectura fácil, el sistema braille y la lengua de signos. Centrándonos en el uso de las reproducciones $3 D$ como herramientas de inclusión social, sobre todo, para las personas con dificultades visuales. El tercer bloque, focalizado en las TIC, se desarrolla en un estudio de fabricación digital en el que se explican las técnicas actuales, y se realiza una práctica de impresión reproduciendo diferentes piezas. El cuarto bloque, se destina a poner en práctica todo lo aprendido: se explica una pieza a través de un texto comprensible, se traduce la explicación en braille y se realiza un taller sobre lengua de signos. Finalmente, las participantes explican la interpretación inclusiva de la obra al resto del grupo clase. Se evalúa la formación por parte del alumnado y el profesorado a través de una evaluación previa, formativa y sumativa. Se analizan los datos bajo una metodología cualitativa utilizando un sistema de
\end{abstract}


categorías, basado en el concepto del patrimonio inclusivo y su apreciación, para describir los cambios conceptuales y actitudinales de las participantes. Los resultados demuestran que, en esta propuesta multidisciplinar realizada por profesionales del ámbito del patrimonio y la digitalización, el alumnado ha sido el sujeto activo de su propio aprendizaje, ha descubierto que el patrimonio es inclusivo y accesible a la diversidad de públicos y ha aplicado lo aprendido realizando una actividad de interpretación inclusiva del patrimonio. Cabe destacar que se han fomentado nuevas vocaciones profesionales. La experiencia ha sido muy positiva, se pretende compartirla con otras profesionales del ámbito y replicar la formación a nivel territorial.

Palabras clave: accesibilidad, adolescentes, fabricación digital, museo, patrimonio inclusivo.

\begin{abstract}
"Looking with our hands", an innovative training education proposal is presented. It aims at understanding the heritage as a tool for learning and social inclusion, through digital fabrication and $3 D$ reproduction. The proposal is structured in four areas. In a first area, secondary school students must choose the most precious they would keep, and fill in a form similar to those used in the inventories of museum collections. This material serves as the basis for the theoretical presentation that, through dialogue and reflection on the selected objects, introduces the basic concepts about the importance of conserving cultural heritage as a pillar of our community identity. In a second area, museum accessibility and communication is at the core. The most vulnerable groups and current resources are explained: easy to read, braille system and sign language. The innovative use of $3 D$ reproduction for social inclusion of visual impaired people is also introduced. The third area, focused on ICT, takes place at a digital fabrication studio. The state of the art techniques are discussed and different pieces are printed. In a fourth area, all learnings are put in practice by explaining a piece through an easy to read text, it is translated in braille and a workshop in sign language is performed. Lastly, participants explain the piece inclusive interpretation to the rest of the class. An initial, formative and summative evaluation is conducted to evaluate students and teachers training. To define conceptual and attitude changes from participants data is analised qualitatively using emerged categories related to inclusive heritage and its appreciation. Results show students have been active subject of their own learning process, have realised inclusive and accessible heritage, and have applied an inclusive interpretation. Remarkably, new professional vocation have been fostered in a very positive experience worth sharing and replicating with other professionals and territories.
\end{abstract}

Keywords: accessibilty, digital fabrication, inclusive heritage, museum, youth. 


\section{Introducción}

El proyecto que presentamos nace cómo un encargo de formación académica para alumnado de Educación Secundaria Obligatoria, que nos animó a iniciar una línea de investigación concreta sobre patrimonio inclusivo y fabricación digital, que se encuentra en fase embrionaria. Concretamente, lo que se pretende investigar es: cómo hacer accesible el patrimonio cultural a adolescentes entre doce y dieciséis años, utilizando la fabricación digital como foco de interés.

El objeto de estudio se centrará en institutos de Educación Secundaria Obligatoria, de la zona metropolitana de Barcelona, que desarrollen proyectos de Servicio Comunitario sobre patrimonio cultural local, y que tengan la posibilidad de realizar un taller de fabricación digital. Las sesiones de formación realizadas que exponemos nos sirven como prueba piloto para desarrollar un futuro trabajo de investigación.

El Servicio de Educación del Ayuntamiento de Santa Perpetua de Mogoda tiene como una de sus líneas de actuación impulsar y desarrollar proyectos de Servicio Comunitario destinados a $3^{\circ}$ y $4^{\circ}$ de ESO, Educación Secundaria Obligatoria. Se trata de una acción educativa que impulsa el Departamento de Educación de la Generalitat de Cataluña, en el marco del currículum obligatorio. Promueve que el alumnado experimente y protagonice acciones de compromiso cívico, aprenda en el ejercicio activo de la ciudadanía, y pongan en juego sus conocimientos y capacidades al servicio de la comunidad.

Dentro de las propuestas para el curso 2020-2021 se impulsó el proyecto 'Mirar con las manos'. Una propuesta de formación innovadora que, en cuatro bloques, pretende dar a conocer el patrimonio local como herramienta de aprendizaje e inclusión social, a través de la fabricación digital y la reproducción 3D. Se realizó una formación de 20h desarrolladas por dos equipos especializados externos: uno en patrimonio cultural y accesibilidad, y otro en fabricación digital.

La motivación para iniciar la investigación posterior parte de una doble necesidad que surge en el diseño de la formación: por un lado, buscar estrategias didácticas para hacer accesible el patrimonio cultural a los adolescentes, y por otro, dar a conocer los nuevos lenguajes emergentes que actualmente están empezando a estar presentes en los museos, y que abren nuevas vocaciones profesionales para los más jóvenes.

Consideramos un tema de gran relevancia la idea preconcebida de que los adolescentes no están interesados en los museos por qué no son accesibles para ellos, que se encuentran barreras que les impiden verlos como espacios culturales a los que acudir en su tiempo libre. La didáctica del patrimonio cultural se presenta como una herramienta de aprendizaje que puede romper estas barreras, y las nuevas tecnologías son un foco de interés de esta franja de edad que permite presentar nuevas profesiones que ayuden a la inclusión social. 


\section{Creación de la formación "Mirar con las manos"}

A continuación, se explica cómo se ha creado la formación.

\subsection{Metodología}

La metodología utilizada en la formación, se ha basado en el diálogo y participación activa por parte de la mayoría del alumnado. Los jóvenes son los protagonistas de su propio proceso de aprendizaje, hecho que ha facilitado el aprendizaje significativo.

Tabla 2. Metodología de la formación "Mirar con las manos"

\begin{tabular}{|l|l|l|l|}
\hline \multicolumn{1}{|c|}{ Contenido de los bloques } & \multicolumn{1}{|c|}{ Profesionales } & \multicolumn{1}{|c|}{ Recursos } & \multicolumn{1}{c|}{ Espacio } \\
\hline $\begin{array}{l}\text { Bloque 1: Los museos y } \\
\text { espacios patrimoniales } \\
\text { ¿hablan de nosotr@s? }\end{array}$ & $\begin{array}{l}\text { Profesionales externas - } \\
\text { patrimonio cultural y } \\
\text { accesibilidad }\end{array}$ & $\begin{array}{l}\text { Activos tradicionales } \\
\text { (presentaciones y } \\
\text { dinámicas participativas) }\end{array}$ & $\begin{array}{l}\text { Aula del centro } \\
\text { educativo }\end{array}$ \\
\hline $\begin{array}{l}\text { Bloque 2: La accesibilidad y } \\
\text { los museos. ¿Qué } \\
\text { entendemos por patrimonio } \\
\text { inclusivo? }\end{array}$ & $\begin{array}{l}\text { Profesionales externas - } \\
\text { patrimonio cultural y } \\
\text { accesibilidad }\end{array}$ & $\begin{array}{l}\text { Activos tradicionales } \\
\text { (presentaciones y } \\
\text { dinámicas participativas) }\end{array}$ & $\begin{array}{l}\text { Aula del centro } \\
\text { educativo }\end{array}$ \\
\hline $\begin{array}{l}\text { Bloque 3: La fabricación } \\
\text { digital y su uso en los } \\
\text { museos. }\end{array}$ & $\begin{array}{l}\text { Profesionales externas - } \\
\text { fabricación digital }\end{array}$ & $\begin{array}{l}\text { Activos TIC } \\
\text { (vectorización 2D e } \\
\text { impresión 3D) }\end{array}$ & Espacio digital \\
\hline $\begin{array}{l}\text { Bloque 4: Valoramos lo que } \\
\text { hemos aprendido y futura } \\
\text { aplicación. }\end{array}$ & $\begin{array}{l}\text { Profesionales externas - } \\
\text { patrimonio cultural y } \\
\text { accesibilidad }\end{array}$ & $\begin{array}{l}\text { Activos tradicionales } \\
\text { (presentaciones y } \\
\text { dinámicas participativas) }\end{array}$ & $\begin{array}{l}\text { Aula del centro } \\
\text { educativo }\end{array}$ \\
\hline
\end{tabular}

Fuente: Elaboración propia

\subsection{Objetivos}

Los objetivos de la formación fueron los siguientes:

- Objetivo 1 (significatividad): Conocer la relación que existe entre patrimonio inclusivo y fabricación digital.

- Objetivo 2 (comunicación): Aprender nuevas formas de comunicación accesible a través de la reproducción de piezas en modelos de impresión 3D.

- Objetivo 3 (acción): Realizar una actividad práctica de interpretación inclusiva del patrimonio.

- Objetivo 4 (proyección): Difundir los resultados de la formación y reconocer su aplicación futura.

Para definir los objetivos nos hemos guiado por el Modelo Competencial Orientador (XTECXarxa Telemàtica Educativa de Catalunya, 2021) que emerge de la orientación educativa 
para favorecer el éxito del alumnado en cuanto a su autonomía y desarrollo de proyecto de vida. Este modelo sigue los criterios que se resumen en el acrónimo SCAP (significatividad, comunicación, acción y proyección). La significatividad hace referencia a todo lo que capta el interés del alumnado, la comunicación se basa en las diferentes formas de expresión, la acción hace referencia a ser activo, a aplicar lo aprendido y finalmente, la proyección se focaliza en cómo puede transferirse lo aprendido fuera del entorno escolar.

\section{Desarrollo de la formación "Mirar con las manos"}

\subsection{Bloque 1: Los museos y espacios patrimoniales, ¿hablan de nosotr@s?}

Se inicia la formación con un primer bloque introductorio sobre conceptos básicos de patrimonio cultural a través de una batería de preguntas, que se utilizan como evaluación previa de conocimientos sobre el tema. En paralelo, el alumnado debe realizar un ejercicio antes de iniciar la formación. Deben seleccionar el objecto que salvarían en caso de catástrofe y rellenar una ficha con las características habituales que recogen los inventarios de los museos.

\subsection{1. ¿Qué saben l@s adolescentes del patrimonio cultural?}

Dentro de la evaluación previa, tres preguntas sencillas nos dieron las claves de la idea preliminar que tenían sobre el patrimonio cultural:

- ¿Cuál es el patrimonio de tú ciudad?

- ¿Crees que hay que protegerlo?

- ¿Por qué sí o por qué no hay que protegerlo?

El alumnado identifica como patrimonio el concepto clásico de grandes monumentos, obras de arte y objetos antiguos. Este concepto de Patrimonio Cultural se inicia a partir de la Revolución Industrial y el Romanticismo cuando se desarrolla la conciencia y la necesidad de preservar las representaciones más destacadas de la cultura humana, generalmente grandes obras de arte y arquitectura (Choay, 2007; Prats, 1998:68). En el texto final de la Conferencia Mundial de la UNESCO (México, 1982) se proponía una definición con una visión más amplia y que en la actualidad es la más aceptada. En ella se incorpora el Patrimonio mueble, inmueble, material e inmaterial. Esta es, a grandes rasgos, la percepción que impera sobre el concepto y es la que se recoge en la normativa legal sobre protección y conservación de patrimonio.

La conciencia que el patrimonio cultural debe protegerse parece clara. Un $90 \%$ responde que sí, y un 10\% que no lo sabe. La clave está en analizar el porqué. Entre las respuestas se repiten ideas, que podemos resumir en tres:

- $\quad$ Porque forman parte de la historia, hablan del pasado. 
- Porque son únicos, hablan de la singularidad de los elementos.

- Porque representan la ciudad, hablan de la identidad compartida.

Utilizando como metodología el diálogo participativo y a través de sus propias respuestas se introducen los contenidos básicos sobre el patrimonio cultural y la importancia de su conservación, siempre utilizando como referentes los propios bienes patrimoniales de cada municipio. Ellos y ellas son los protagonistas de su propio aprendizaje.

\subsection{2. ¿Qué explican los objetos de los museos de nosotr@s?}

El museo sigue viéndose como el lugar donde se guardan los objetos patrimoniales. Igual que con el concepto de patrimonio cultural, en el imaginario colectivo sigue imperando la imagen tradicional de museo. En la evaluación previa se destinaban tres preguntas para detectar estos conceptos previos:

- ¿Has visitado alguna vez el museo de tu ciudad?

- ¿Con quién lo has visitado? Escuela, Familia, Amigos, Otros.

- ¿Crees que es un espacio que habla de tus intereses?

- Indica 3 temas que te gustaría encontrar en un museo.

El $90 \%$ del alumnado encuestado había visitado el museo de su ciudad con la escuela o instituto, y un $10 \%$ también con la familia. A la pregunta si el museo habla de sus intereses, esperábamos una respuesta clara de 'NO', limitadas como indicábamos en la introducción por ideas preconcebidas sobre la franja de edad. Los resultados fueron: 20\% Sí, el 30\% No y un 50\% no lo sé. Los temas que les gustaría encontrar en los museos fueron: objetos, cultura, historia, prehistoria, mitología, música, arte, ciencia, imágenes antiguas y espacios diferentes. $\mathrm{Y}$ en este punto empezamos a trabajar analizando y reflexionando a partir de los objetos que cada uno salvaría en caso de catástrofe.

Dentro del conjunto presentado en las fichas podemos hablar de objetos con significado personal mayoritariamente, asociados a antepasados y que transmiten recuerdos de momentos o personas. Nos encontramos con: peluches, cajas decorativas, aparatos de música, libros y también, los móviles y la Play Station. 

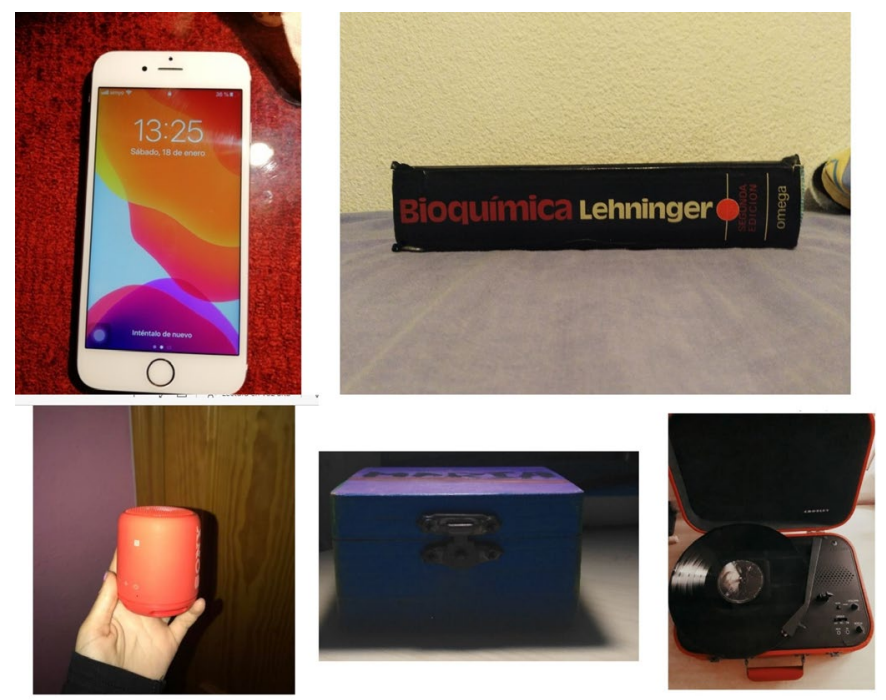

Fuente: Elaboración propia

Fig. 1 Composición de los objetos seleccionados por el alumnado

Cada persona explicaba el objeto seleccionado y la historia que lleva asociado. Nos encontramos con objetos utilitarios, decorativos, científicos y también simbólicos. Objetos que están muy relacionados con los intereses y temas que habían seleccionado previamente.

La reflexión se orientaba a poner el foco de interés en los museos, los espacios en los que los objetos nos hablan de nuestra cultura y nuestra historia compartida. Las características básicas que se recogen en la ficha de inventario: la fecha, el material, las medidas, etc. son muchas veces, las que acompañan a los objetos en los museos, pero no suele ser este tipo de datos los que interesan a los visitantes no especialistas. Tan sólo un alumno construyó un relato, una historia, para explicar su objeto, y nos dio el punto de partida para introducir el tema de la didáctica del patrimonio y los nuevos lenguajes digitales.

\subsection{3. ¿Los museos hablan el lenguajedel@s jóvenes?}

La didáctica del patrimonio es un conjunto de técnicas destinadas a proporcionar la comprensión o interpretación del patrimonio a la mayoría de la ciudadanía. Los objetos deben explicarnos historias que nos lleguen, que despierten la curiosidad, que nos hagan emocionar. Sin emoción no hay aprendizaje, y aquí es donde empezamos a reflexionar sobre si los museos le parecen lugares próximos a su realidad.

Los lenguajes museográficos tradicionales se limitaban a: textos, cartelas, imágenes y audiovisuales, pero actualmente encontramos que poco a poco los recursos digitales y las nuevas tecnologías van estando presentes en los museos. Vivimos un proceso de cambio en la definición de lo qué es un museo y para qué sirve, pero la tecnología sin didáctica nos situará igual de lejos de los adolescentes, si no partimos de sus intereses. En su tiempo libre 
utilizan todo tipo de recursos tecnológicos que se basan en la interacción y la participación. Los museos siguen siendo espacios más de mirar que de actuar, espacios pasivos en los que no se sienten protagonistas.

En el cuestionario previo tenían que indicar tres palabras que definieran sus experiencias visitando museos. Partíamos de diferentes estudios publicados sobre la percepción del patrimonio cultural de los jóvenes (Becerra y Domínguez, 2014; Santacana et al. 2016) y esperábamos encontrar la palabra 'Aburrida' en primer término, pero no fue así. Hasta el momento, las respuestas como se ve en la nube de palabras, son positivas. Destacan dos palabras a nuestro parece claves: interesante y curiosa.

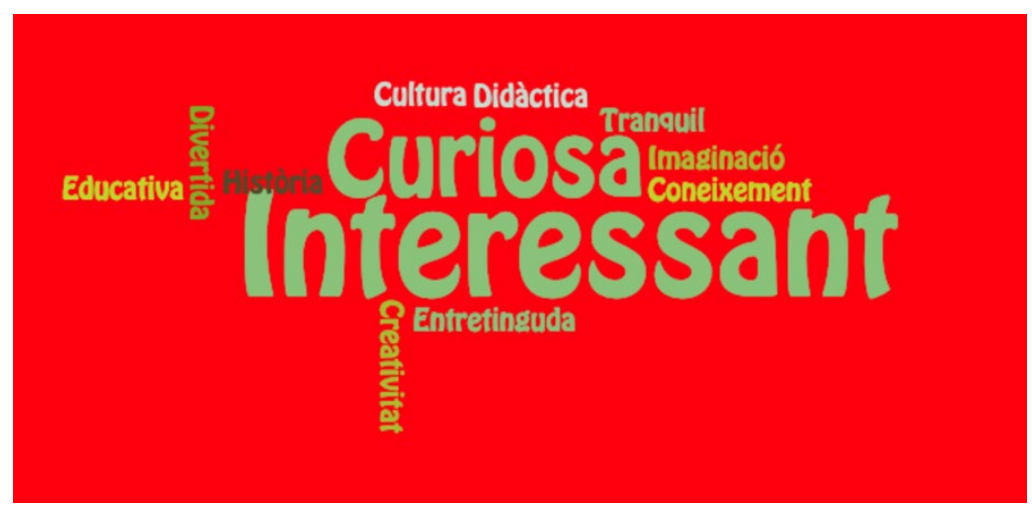

Fuente: Elaboración propia

Fig. 2 Palabras que describen la experiencia del alumnado visitando museos

\subsection{Bloque 2: La accesibilidad en los museos}

En el segundo bloque se parte del marco conceptual, se explica qué entendemos por accesibilidad: los productos y los servicios son accesibles en la medida que todas las personas somos capaces de utilizarlos. Se enfatiza que es una condición importante para garantizar la autonomía, la no discriminación y la igualdad de oportunidades para todas las personas.

A continuación, se incide en cómo podemos trasladar esta temática en el ámbito de los museos, que se traducirá en beneficio para todas las personas. Se pregunta al alumnado si creen que todas las personas tienen acceso a nuestro patrimonio y se reflexiona acerca de cómo es el público que visita los equipamientos culturales: familias, grupos escolares, jóvenes, personas adultas, gente mayor y personas con diversidad funcional, "personas que por motivos de la diferencia de funcionamiento de su cuerpo realizan las tareas habituales (desplazarse, comunicarse, etc.) de manera diferente" (Romañac y Lobato, 2005).

También se hace referencia al acceso digital, al público virtual que visita el museo a través de la página web, por ejemplo, disfrutando de una visita virtual del mismo, o realizando algún tipo de actividad on-line propuesta por el museo. Se destaca que, en muchas ocasiones, cuando se facilitan actividades a través de vídeos on-line por parte del museo, estos recursos 
digitales no son accesibles, con lo cual, los colectivos con más dificultades, siguen siendo excluidos.

El objetivo es traspasar la connotación negativa de "la discapacidad", destacar la variedad y no la diferencia y, sobre todo, poner la persona en primera instancia. Se ponen ejemplos sobre condiciones de diversidad permanentes, temporales y situacionales referente a los cinco sentidos: tocar, ver, escuchar y hablar para que el alumnado pueda constatar, que se pueden tener necesidades específicas a lo largo de toda la vida y que los recursos de accesibilidad serán beneficiosos para todas las personas.

\subsection{1. ¿Qué barreras podemos encontrar en un equipamiento cultural?}

Se explican las barreras que nos podemos encontrar en un equipamiento patrimonial: las arquitectónicas, barreras de carácter físico que limitan o impiden la interacción de las personas con el entorno, las de comunicación, barreras que limitan o impiden la expresión y la recepción de la información, y las de actitud, barreras que generan una acción discriminatoria, seguramente una de las más difíciles de superar.

A continuación, se da a conocer el concepto del "Diseño universal o para todas las personas" entendido como: el diseño de entornos, espacios, edificios, servicios, medios de transporte, productos y dispositivos que garantizan que, sin que sean necesarias las adaptaciones, todas las personas pueden acceder. Seguidamente, se explican los siete principios de diseño universal: igualdad de uso, flexible, simple e intuitivo, información fácil de percibir, con tolerancia al error, poco esfuerzo físico y dimensionado adecuado.

La formación se centra en el uso de las reproducciones 3D como herramientas de inclusión social, sobre todo, para las personas con dificultades visuales. El alumnado aprende que las reproducciones $3 \mathrm{D}$ de piezas del patrimonio local pueden servir como recursos táctiles útiles para todas las personas ya que incluyen la novedad, la vista y el tacto.

Se trabaja con el alumnado sobre todo la accesibilidad en la comunicación, a través de los siguientes recursos: la lectura fácil, se dan nociones básicas para aprender a escribir un texto de la manera más comprensible posible, se da a conocer el sistema braille y se facilita su alfabeto, y se realiza un taller sobre la lengua de signos para que el alumnado pueda aprender las nociones básicas de esta lengua.

\subsection{Bloque 3: La fabricación digital y su uso en los museos}

El tercer bloque, focalizado en la tecnología 4.0, se desarrolla en un estudio de fabricación digital en el que se explican las técnicas actuales, y se realiza una práctica de impresión reproduciendo diferentes piezas. Este bloque se realizó en el Espai SPMakers que el Ayuntamiento de Santa Perpetua de Mogoda y AMERC Asociación de Municipios del Eje de la Riera de Caldes han puesto en marcha con tres objetivos: acelerar la adopción de estrategias y la implantación de tecnologías 4.0 en las empresas; facilitar la formación, el reciclaje y la especialización de profesionales, y sensibilizar y promover las vocaciones industriales entre las jóvenes, las personas desocupadas y la ciudadanía. 
A partir de la maquinaria disponible actualmente en el espacio Makers, el alumnado tuvo la oportunidad de trabajar varios aspectos.

\subsubsection{Vectorización digital de elementos $2 D$}

A partir del dibujo arqueológico de la inscripción íbera de la Estela, extrajeron los grafitis para ser reproducidos sobre madera a través de la cortadora láser. Produjeron por tanto un prototipo táctil, respetando la forma original de la pieza.

Esta misma vectorización también la imprimieron en vinilo, y pusieron en práctica el proceso de traspaso del vinilo a la superficie de exposición. Este es un recurso habitual en la rotulación de museos, pero también en comercios y espacios públicos. Ambos materiales, permitirían a una persona con déficit visual "leer" la inscripción de la pieza y analizar los signos del alfabeto íbero que la configuran.

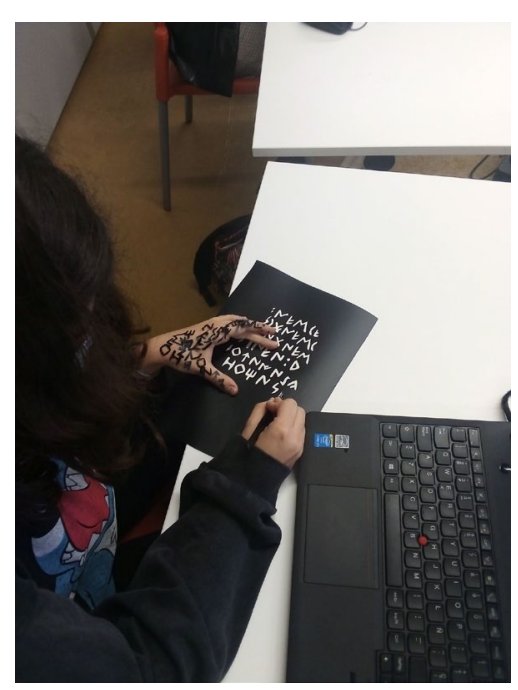

Fig. 3 Taller de vectorización 2D en Espai SPMakers

Para contextualizar el uso de esta técnica en museos, se explicó como ejemplo los planos táctiles que pueden encontrarse en el Vilamuseo de Villajoiosa (Alicante) o los dosieres de visita con láminas en relieve del Programa La Mirada Táctil presentes en la Red de Museos Locales de la Diputación de Barcelona.

\subsubsection{Impresión $3 D$}

El alumnado también experimentó con el escaneo 3D y su posterior impresión 3D. En este caso, a partir de un modelo 3D a escala de toda la pieza, vieron cómo se genera la impresión. Asimismo, practicaron con el escáner 3D y la modelización a partir de sus propias figuras. 

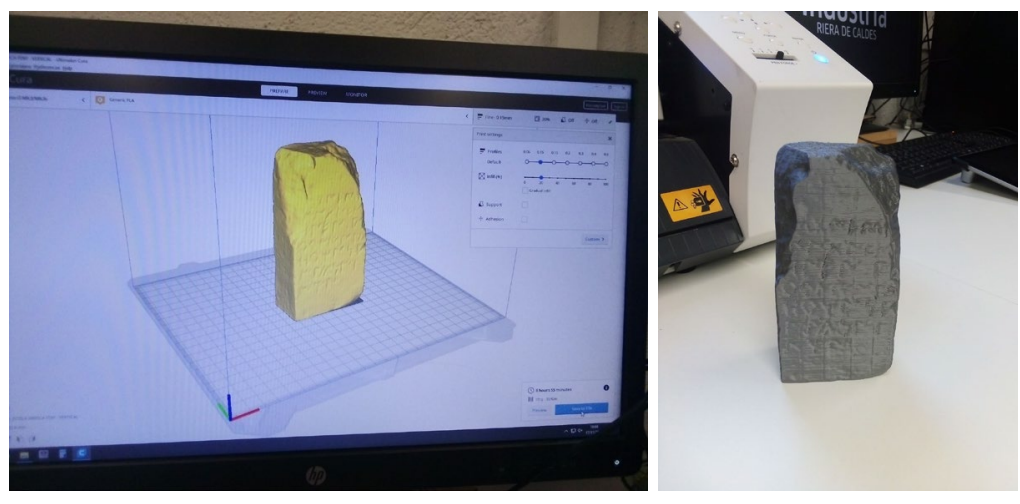

Fuente: Blog Santa Perpetua Educa

Fig. 4 Taller de impresión 3D en Espai SPMakers

En Cataluña, a través del Departamento de Patrimonio Cultural de la Generalitat, se ha realizado diferentes experiencias con la impresión en 3D en diferentes áreas: documentación, restauración, investigación y difusión. El más reciente es el programa Giravolt que va más allá de la publicación en línea de los modelos 3D. Es un proyecto integral que promociona el uso de la tecnología del escaneo 3D dentro del sector del patrimonio cultural catalán. Una iniciativa colaborativa y transversal que aporta un nuevo formato para la conservación y la divulgación de la cultura. Ya se han creado más de 100 modelos $3 \mathrm{D}$ del patrimonio cultural catalán que pueden consultarse en abierto a través del canal de Sketch Fab.

\subsection{Bloque 4: Valoramos lo que hemos aprendido y aplicación práctica}

Finalmente, se destina un espacio de la formación a que el alumnado pueda expresar lo que ha aprendido para posteriormente, poder transferir los conocimientos adquiridos a través de acciones de servicio a la comunidad. Se trata de ofrecer nuevos recursos inclusivos y accesibles para interpretar el patrimonio local. Como hemos dicho, sin emoción no hay aprendizaje, en este caso, el alumnado constata su emoción al ver funcionar las impresoras 3D y valoró positivamente la formación.

Posteriormente a la formación, las participantes se dividen en tres grupos para diseñar actuaciones específicas de interpretación del patrimonio local a través de los recursos de accesibilidad explicados. El primer grupo escribe un texto comprensible sobre la pieza patrimonial escogida, hecho que refleja lo aprendido respecto a la lectura fácil. El segundo grupo traduce, el texto previamente redactado sobre la pieza del patrimonio local, a braille. Finalmente, el tercer grupo hace un vídeo audio guiado explicando el contenido en lengua de signos. Tanto la ficha técnica en Braille como el video son accesibles a través de códigos QR. Todos los grupos expusieron sus actuaciones al resto del grupo clase. 


\subsubsection{Visita al Museo de Arqueología de Cataluña (Barcelona)}

El alumnado de la formación "Mirar con las manos" del Instituto Estela Ibérica visitó la exposición "El Enigma Íbero" del Museo de Arqueología de Cataluña el pasado 28 de mayo de 2021, donde se expone la pieza original de la Estela Ibérica que trabajaron en las diferentes sesiones de formación y que da nombre al instituto.

El propio museo invitó al alumnado para conocer los detalles de las actuaciones de interpretación inclusiva del patrimonio. El alumnado presentó su trabajo y entregó al museo una copia de todos los materiales creados para que puedan servir de apoyo a las actividades divulgativas. El equipamiento cultural se ha ofrecido a dar continuidad al proyecto durante el próximo curso 2021-2022 para poder añadirlo a las actividades de su programa educativo y dirigirlo a las personas con diversidad funcional.

\section{Resultados}

Los resultados que se presentan a continuación, a partir de ejemplos de las narrativas de los sujetos de investigación, muestran la consecución de los objetivos planteados.

\subsection{Significatividad}

"Conocer la importancia del patrimonio, lo que se puede hacer con él y el conocimiento de las nuevas alternativas para las personas con diversidad funcional” (Sujeto 7).

Las observaciones realizadas de las sesiones demuestran que a lo largo de la formación "Mirar con las manos" se estableció diálogo y participación activa por parte de la mayoría del alumnado participante. También se constata que se ha partido de la realidad y los intereses del alumnado, hecho que ha facilitado el aprendizaje significativo.

De las respuestas de los cuestionarios se extrae que, lo que ha captado el interés tanto del alumnado como del profesorado, es conocer el patrimonio local, y ver que éste es inclusivo y accesible a la diversidad de públicos. En general, lo que más ha gustado al alumnado, es la novedad de experimentar cómo hacer una reproducción 3D de una pieza del patrimonio local. Lo que revelan que se podría mejorar, es la presencialidad de todas las sesiones.

Cabe mencionar que el hecho de salir del aula y que el alumnado pudiera trasladarse al espacio digital supuso un nuevo espacio de aprendizaje donde se dio la igualdad de oportunidades para aprender por parte de todo el alumnado, que mayoritariamente desconocía esta tecnología digital. Poder acudir al espacio de fabricación digital en la situación actual de pandemia se tradujo en alivio y motivación para las participantes del grupo clase que previamente había estado confinado.

\subsection{Comunicación}




\section{“Descubrir todas las posibilidades que tiene el 3D” (Sujeto 9).}

Después de la formación, el alumnado constató que han aprendido diferentes formas de expresión e interpretación del patrimonio. La reproducción 3D permite jugar con la escala e implica la posibilidad de la accesibilidad táctil, todas las personas pueden tocar la reproducción de la pieza y percibir cómo es el objeto original. El éxito de utilizar las TIC en los contextos educativos reside en: la sencillez, la usabilidad, el diseño atractivo, la adaptabilidad a las necesidades y la accesibilidad.

\subsection{Acción}

“Ver las máquinas 3D y aprender cómo funcionan” (Sujeto 8).

Las participantes valoraron positivamente las sesiones de formación y confirmaron que les habían ayudado a realizar las actuaciones específicas de interpretación del patrimonio local. Se valora muy positivamente lo que el alumnado cita como "la excursión al espacio digital" y la aplicación práctica de lo aprendido.

Todas las participantes recomendarían realizar esta formación a otras compañeras y a la mayoría les gustaría seguir aprendiendo sobre las posibilidades que tiene el 3D en el ámbito patrimonial. Se considera una formación efectiva ya que todas las participantes consideran que han aprendido aspectos del patrimonio que desconocían.

\subsection{Proyección}

“Incluir la tecnología para hacer más dinámicas las visitas a los centros culturales" (Sujeto 10).

El alumnado transfirió el aprendizaje adquirido cuando, a través de una invitación por parte del Museo de Arqueología de Cataluña, pudo visitar el museo y presentar, al director y al técnico de educación, las actuaciones de interpretación inclusiva del patrimonio trabajadas.

El hecho de que el equipamiento cultural se haya ofrecido a dar continuidad al proyecto y añadirlo a sus actividades ha empoderado al alumnado quien se ha sentido capaz y útil al ver traducido su proyecto de Servicio Comunitario en una acción real del museo dirigida a las personas con diversidad funcional.

Otro aspecto relevante es que se han fomentado nuevas vocaciones profesionales tanto en el ámbito del patrimonio, de la digitalización como incluso profesiones relacionadas con el ámbito de la accesibilidad. Un claro ejemplo, es el caso de un alumno que comentó que había descubierto su vocación, aprender lengua de signos para ser intérprete. 


\section{Conclusiones}

En conclusión, la experiencia formativa ha sido muy positiva. El objetivo es compartir la propuesta "Mirar con las manos" con otras profesionales del ámbito y que se pueda replicar a nivel territorial. La participación dentro del proyecto de Servicio Comunitario de dos equipos especializados externos: uno en patrimonio cultural y accesibilidad, y otro en fabricación digital ha permitido incorporar las TIC como modelo de enseñanza y aprendizaje a través de herramientas accesibles de interpretación patrimonial. Los resultados demuestran que se ha conseguido la proyección de los conocimientos adquiridos, ya que el alumnado ha aplicado lo aprendido a una actividad concreta de interpretación inclusiva del patrimonio. Se trata de una prueba piloto que sienta las bases para diseñar un proyecto de investigación más amplio que permita el acceso de los más jóvenes al patrimonio cultural.

\section{Referencias}

BECERRA MUÑOZ, E. y DOMINGUEZ CONTRERAS, B. (2014). Museos, comunicación y jóvenes: la comunicación y sus efectos en la población de referencia del museo. Historia y Comunicación Social, 19, p.603-611.

CHOAY, F. (2007). Alegoría del patrimonio. Barcelona: Gustavo Gili.

PRATS, L. (1998). El concepto de patrimonio cultural. Politica y Sociedad, 27, p. 63-76.

SANTACANA MESTRE, J. et al. (2016). ¿Qué opinan los adolescentes sobre los museos y la didáctica? en Didáctica de las Ciencias Experimentales y Sociales, 31, p. 23-38.

ROMAÑAC, J. y LOBATO, M. (2005). Diversidad funcional, nuevo término para la lucha por la dignidad en la diversidad. $<$ http://forovidaindependiente.org/wpcontent/uploads/diversidad_funcional.pdf $>$ [Consulta: 25 de febrero de 2021]

UNESCO (1982). Declaración de México sobre las políticas culturales. México DF.

XTEC - Xarxa Telemàtica Educativa de Catalunya (2021). Model Competencial Orientador. $<$ http://xtec.gencat.cat/ca/curriculum/orientacioeducativa/model-competencial-orientador/\#> [Consulta: 25 de febrero de 2021] 